\title{
Das obstruktive Schlafapnoe-Syndrom (OSAS) - Therapie
}

\section{B. Sanner ${ }^{1}$ \\ C. Hader ${ }^{2}$ \\ K. Rasche ${ }^{2}$}

\section{Obstructive sleep apnea syndrome - therapy}

Die Indikation zur Einleitung einer Therapie bei Nachweis eines obstruktiven Schlafapnoe-Syndroms ist immer dann gegeben, wenn die erhobenen Befunde der vorangegangenen Diagnostik die Beschwerdesymptomatik der Patienten erklären. Dies kann auch Patienten betreffen, die zwar nur einen niedrigen Apnoe-/Hypopnoe-Index (AHI) haben, in Folge eines hohen respiratorischen Weckindex (Arousalindex) jedoch eine ausgeprägte Tagesmüdigkeit aufweisen. Die alleinige Orientierung am Apnoe-/HypopnoeIndex oder an der Sauerstoffsättigung ist nicht gerechtfertigt.

Ob andererseits die Therapie eines asymptomatischen Patienten mit einem schweren Befund notwendig ist, um kardiovaskuläre Komplikationen zu verringern, wird z.Zt. kontrovers diskutiert (5). Auch unabhängig von diesem theoretischen Vorteil sollte bei diesen Patienten aber ein Behandlungsversuch großzügig durchgeführt werden: Viele Patienten mit hohem Apnoe-/Hypopnoe-Index oder deutlich gestörter Schlafarchitektur realisieren ihre subjektive Beeinträchtigung erst nach Besserung ihres Befindens unter Therapie.

Ziel einer Behandlung der obstruktiven Schlafapnoe ist die möglichst vollständige Beseitigung der schlafbezogenen Atmungsstörung, also der pharyngealen Obstruktion, und damit die Verhinderung von Apnoen, Hypopnoen, Schnarchen und vermehrten intrathorakalen Druckschwankungen mit konsekutiven Weckreaktionen. Dies sollte angestrebt werden in allen Körperpositionen also vor allem in Rückenlage - und in allen Schlafstadien - also vor allem auch im REM-Schlaf. Im REM-Schlaf treten nämlich aufgrund des niedrigen Muskeltonus, der mit diesem Schlafstadium assoziiert ist, besonders häufig pathologische respiratorische Ereignisse auf.

\section{Konservative Therapie}

\section{Allgemeinmaßnahmen}

Grundsätzlich sollten alle Patienten mit nachgewiesener obstruktiver Schlafapnoe Allgemeinmaßnahmen berücksichtigen. Hierzu zählt bei übergewichtigen Patienten die Gewichtsabnahme, da hierbei parallel zur Reduktion des Körpergewichts eine Abnahme des parapharyngealen Fettgewebes und damit eine Erweiterung der extrathorakalen Atemwege mit konsekutiver Besserung des Befundes erzielt werden kann. Zusätzlich sollten Alkohol oder Sedativa, die den Muskeltonus während des Schlafes reduzieren können, gemieden werden. Allgemeine Regeln der Schlafhygiene wie das Einhalten regelmäßiger Schlaf/Wachzeiten, Schlaf in einem ruhigen Umfeld und Vermeiden von Schlafentzug - können sich ebenfalls günstig auswirken.

Insgesamt bewirken diese Allgemeinmaßnahmen bei symptomatischen Patienten in der Regel zwar nur eine geringe Befundbesserung, können aber im Einzelfall zu einem Verschwinden der obstruktiven Schlafapnoe führen.

Liegt eine streng lageabhängige obstruktive Schlafapnoe vor - diese besteht dann meist in Rückenlage -, kann über ein Lagetraining mit seitlicher Schlafposition eine Besserung erzielt werden.

\section{Medikamentöse Therapie}

In der Vergangenheit wurden zur Behandlung der obstruktiven Schlafapnoe eine Vielzahl von medikamentösen Substanzen eingesetzt. Hierzu zählten Analeptika, Antidepressiva, Carboanhydrasehemmer, Methylxanthine oder Opiatantagonisten. Für keine dieser Substanzen konnte jedoch ein positiver Effekt nachgewiesen werden, eine medikamentöse Therapie zur alleinigen Behandlung der obstruktiven Schlafapnoe sollte daher nicht durchgeführt werden (6).

Institut

${ }^{1}$ Bethesda-Krankenhaus, Akademisches Lehrkrankenhaus der Ruhr-Universität Bochum, Medizinische Klinik, Wuppertal

2 Kliniken St. Antonius, Akademisches Lehrkrankenhaus der Heinrich Heine-Universität Düsseldorf,

Zentrum für Innere Medizin - Schwerpunkt Pneumologie

Korrespondenz

Prof. Dr. med. B. Sanner - Bethesda Krankenhaus, Akad. Lehrkrankenhaus der Ruhr-Universität Bochum Medizinische Klinik · Hainstraße 35 · 42109 Wuppertal · Tel.: 0202/2902002 · Fax: 0202/2902005 .

E-Mail: bernd.sanner@bethesda-wuppertal.de

eingereicht: 3.12 .2003 - akzeptiert: 18.2 .2004

Bibliografie

DOI: $10.1055 / \mathrm{s}-2004-820547$

Dtsch Med Wochenschr 2004; 129:570-572 • @ Georg Thieme Verlag Stuttgart · New York · ISSN 0012-0472 
kurzgefasst: Bei allen Patienten mit dokumentierter obstruktiver Schlafapnoe sollten Faktoren gemieden oder reduziert werden, die die schlafbezogene Atmungsstörung verstärken können. Eine medikamentöse Therapie der obstruktiven Schlafapnoe gibt es z.Zt. nicht. Bei Adipositas sollte eine Gewichtsreduktion angestrebt werden.

\section{Apparative Therapie}

\section{Intraorale Hilfsmittel}

Intraorale Hilfsmittel wirken meist über eine Vorverlagerung des Unterkiefers mit dem Ziel, die extrathorakalen Atemwege zu erweitern (14). Die Effektivität intraoraler Hilfsmittel ist der Standardtherapie der obstruktiven Schlafapnoe mit kontinuierlichem positiven Atemwegsdruck (continuous positive airway pressure, CPAP) (s.u.) signifikant unterlegen, wird von einigen Patienten aber gerade im Vergleich zur (Be)atmungstherapie wegen der geringeren lokalen Beeinträchtigung präferiert (4).

Intraorale Hilfsmittel können dann zum Einsatz kommen, wenn die etablierte Therapie mit CPAP ineffektiv war oder vom Patienten abgelehnt wurde. Möglicherweise gibt es Subgruppen von Schlafapnoe-Patienten, die von einer Therapie mit einem intraoralen Hilfsmittel profitieren. Effektive Prädiktoren, die den Therapieerfolg voraussagen, sind jedoch noch nicht bekannt.

\section{Sauerstofftherapie}

Bei Patienten mit reiner obstruktiver Schlafapnoe ist eine nächtliche Sauerstofftherapie nicht indiziert, ggf. kann sie bei Patienten mit koexistenter chronisch obstruktiver Atemwegserkrankung zusätzlich eingesetzt werden.

\section{CPAP-Therapie}

Seit Einführung von CPAP in die klinische Routine im Jahre 1981 durch Sullivan et al. hat sich diese Behandlungsform zur StandardTherapie der obstruktiven Schlafapnoe entwickelt (17). Dies ist nicht zuletzt darauf zurückzuführen, dass sich diese Therapie durch eine hohe Effektivität auszeichnet. Bei mehr als 90\% der Patienten mit obstruktiver Schlafapnoe gelingt mittels CPAP eine Normalisierung der Atmung im Schlaf (1).

Bei der CPAP-Therapie wird über eine Nasenmaske oder eine Nasen-Mund-Maske ein kontinuierlicher positiver Druck in den Atemwegen aufgebaut, um den negativen intrapharyngealen Drucken, die zum Kollaps der oberen Atemwege führen, entgegenzuwirken und die Atmung im Schlaf zu normalisieren.

Die Einstellung auf eine CPAP-Therapie muss unter polysomnographischer Kontrolle erfolgen; denn nur mit Hilfe der Polysomnographie kann erkannt werden, dass sich nicht nur Sauerstoffsättigung und Atmung - gemessen als Luftfluss an Nase und Mund - normalisieren, sondern dass auch die Zahl der respiratorisch bedingten Weckreaktionen reduziert und die Schlafstruktur normalisiert wird.

Ergebnisse randomisierter Studien konnten aufzeigen, dass durch CPAP eine substanzielle Verbesserung neurokognitiver Funktionen und der Lebensqualität erzielt werden kann. Außerdem nimmt die Tagesmüdigkeit ab $(7,15)$. Dies gilt nicht nur für Patienten mit einer schweren obstruktiven Schlafapnoe; auch bei Patien- ten mit nur leicht ausgeprägter Erkrankung hat sich CPAP in kontrollierten und randomisierten Studien als effektiv und wirksamer als andere Therapieformen erwiesen (4).

Zusätzlich bewirkt die konsequent durchgeführte CPAP-Therapie eine Senkung des Blutdruckes (10), vor allem bei prätherapeutisch hypertensiven Patienten mit indirekten Zeichen der Sympathikusaktivierung, also hoher Herzfrequenz in Ruhe und hohem Pulsdruck (16). Auch andere kardiovaskuläre Parameter werden günstig beeinflusst: So kann sich bei eingeschränkter linksventrikulärer Ejektionsfraktion diese unter Therapie bessern (9), oder die gestörte Endothelfunktion kann sich wieder normalisieren (3).

Lokale Nebenwirkungen können in bis zu 50\% der Fälle auftreten, zwingen in der Regel aber nicht zu einem Abbruch der Therapie (12). In einzelnen Fällen kann es notwendig werden, nasale Symptome durch Luftbefeuchter oder nasal applizierte Steroide zu behandeln. Die Compliance liegt nach einjähriger Beobachtungszeit im Mittel bei $70-80 \%$ und ist damit im Vergleich zur medikamentösen Therapie bei internistischen Erkrankungen als hoch einzustufen.

\section{Auto-PAP-Therapie}

Bei der konventionellen CPAP-Therapie wird der Druck manuell eingestellt, der notwendig ist, um in allen Schlafstadien und in allen Körperpositionen die extrathorakalen Atemwege offen zu halten. Dieser Druck bleibt während der gesamten Schlafzeit konstant. Nur wenige Patienten haben jedoch einen kontinuierlich gleichen Druckbedarf, um die Atemwege offen zu halten. So kann der Druckbedarf nicht nur innerhalb der Nacht je nach Schlafstadium und Körperposition, sondern auch kurzfristig durch z.B. Schlafentzug oder eine nasale Obstruktion und langfristig durch Änderung des Körpergewichtes variieren.

Im Unterschied zu den konventionellen CPAP-Systemen werden bei der autotitrierenden PAP-Therapie kontinuierlich entweder pneumotachographisch eine Drucklimitation oder oszillometrisch eine Änderung des Widerstandes der oberen Atemwege erfasst und konsekutiv eine automatische Druckanpassung vorgenommen $(11,18)$. Dies bedeutet, dass sich der maschinell applizierte Druck während der gesamten Nacht ändern kann und intraindividuell auch von Tag zu Tag - je nach Körperposition, Schlafstadien oder anderen prädisponierenden Faktoren - unterschiedliche Druckverläufe dokumentiert werden können.

Autotitrierende Systeme scheinen insbesondere für die Patienten vorteilhaft, die nur kurzzeitig (z.B. während des REM-Schlafes oder in Rückenlage) pathologische Atemmuster während des Schlafes aufweisen. Die Langzeit-Effektivität bei der Behandlung mit einem autotitrierenden System ist der eines konventionellen CPAP-Systems vergleichbar, der mittlere applizierte Druck in den meisten Studien niedriger (2). Möglicherweise bewirkt dies bei einem Teil der Patienten eine bessere Langzeit-Compliance (8).

\section{Bilevel-PAP-Therapie (n-BiPAP)}

Bilevel-PAP-Geräte ermöglichen, den inspiratorischen und exspiratorischen Atmungsdruck jeweils getrennt zu regulieren. In der Regel handelt es sich um druckgetriggerte Geräte, welche sich der Atmung des Patienten anpassen. Bilevel-PAP-Geräte und CPAP-Geräte weisen in der Behandlung der obstruktiven Schlafapnoe die 
gleiche Effektivität auf (13). Eine Indikation für Bilevel-Geräte kann bei den Patienten gegeben sein, die hohe CPAP-Drucke benötigen und durch die erschwerte Ausatmung beeinträchtigt sind. Dies kann der Fall sein, wenn der erforderliche Beatmungsdruck sehr hoch ist oder eine obstruktive Atemwegserkrankung vorliegt.

\section{Nachuntersuchungen}

Nach Einleitung einer konservativen oder apparativen Therapie sollte der Erfolg innerhalb von 3-6 Monaten kontrolliert werden. Die erste Kontrolluntersuchung erfolgt in der Regel polysomnographisch, weitere Nachuntersuchungen können polygraphisch (ohne Aufzeichnung des Schlafes) und ambulant durchgeführt werden, wenn zwischenzeitlich keine substanzielle Gewichtsänderung stattgefunden und die klinische Symptomatik sich nicht verschlechtert hat, insbesondere keine wesentliche Tagesmüdigkeit besteht.

\section{kurzgefasst: Die CPAP-Therapie ist die Standard-Therapie} der obstruktiven Schlafapnoe mit hoher Effektivität. Unter konsequent durchgeführter CPAP-Therapie bessern sich nicht nur die Lebensqualität und Tagesbefindlichkeit, sondern es können auch kardiovaskuläre Parameter, wie eine arterielle Hypertonie, günstig beeinflusst werden. Dies rechtfertigt auch einen Therapieversuch mit CPAP bei Patienten mit relevantem Apnoe-Befund während des Schlafes und einer nur geringen klinischen Beschwerdesymptomatik.

\section{Chirurgische Therapie}

Liegt eine eindeutig behebbare Ursache der obstruktiven Schlafapnoe vor, z. B. in Form von anatomischen Deformitäten wie einer Tonsillenhyperplasie oder Trachealstenose, kann die operative Behandlung zu einer Normalisierung der Atmung während des Schlafes führen.

Die Indikation zu darüber hinausgehenden chirurgischen Verfahren ist sehr viel kritischer zu stellen, da für die meisten dieser Therapien Prädiktoren der Effektivität fehlen. Zum Einsatz kommen die Uvulopalatopharyngeoplastie, die laserassistierte Uvulopalatoplastie, die partielle Resektion von Teilen der Zunge oder auch die Rekonstruktion und Vorverlagerung der Mandibula. Die operativen Eingriffe können nicht nur mit Komplikationen behaftet sein, sondern sind auch meist irreversibel. Bei einer Untergruppe von Patienten mit kraniofazialen Malformationen, also z.B. angeborenen oder erworbenen Kieferfehlstellungen wie der Retrogenie, können plastische Kieferoperationen sehr erfolgreich sein.

kurzgefasst: Liegt eine behebbare anatomische Ursache der obstruktiven Schlafapnoe vor, sollte die Indikation zur operativen Therapie geprüft werden. Die Uvulopalatopharyngeoplastie sollte nicht mehr durchgeführt werden.
Literatur

${ }^{1}$ Becker H, Stammnitz A, Schneider H, Peter JH, von Wichert P. Nasale CPAP Therapie bei obstruktiver Schlafapnoe. Dtsch Med Wochenschr 1995; 120: 783-789

2 Berry RB, Parish JM, Hartse KM. The use of auto-titrating continuous positiv airway pressure for treatment of adult obstructive sleep apnea. Sleep 2002; 25: 148-173

3 Duchna HW, Guilleminault C, Stoohs RA et al. Vascular reactivity in obstructive sleep apnea syndrome. Am J Respir Crit Care Med 2000; 161: 187-191

${ }^{4}$ Engleman HM, Kingshott RN, Wraith PK, Mackay TW, Deary IJ, Douglas NJ. Randomized placebo-controlled crossover trial of continuous positive airway pressure for mild sleep apnea/hypopnea syndrome. Am J Respir Crit Care Med 1999; 159: 461-467

${ }^{5}$ Hedner J, Grote L. The link between sleep apnea and cardiovascular disease: time to target the nonsleepy sleep apneics? Am J Respir Crit Care Med 2001; 163: 5-6

${ }^{6}$ Hein H, Magnussen H. Wie steht es um die medikamentöse Therapie bei schlafbezogenen Atmungsstörungen? Somnologie 1998; 2: 77-88

7 Jenkinson C, Davies RJ, Mullins R, Stradling JR. Comparison of therapeutic and subtherapeutic nasal continuous positive airway pressure for obstructive sleep apnoea: a randomised prospective parallel trial. Lancet 1999; 353: 2100-2105

${ }^{8}$ Konermann M, Sanner B, Vyleta M, Laschewski F, Grötz J, Sturm A, Zidek $W$. Use of conventional and self-adjusting nasal continuous positive airway pressure for treatment of severe obstructive sleep apnea syndrome. Chest 1998; 113: 714-718

9 Laaban JP, Pascal-Sebaoun S, Bloch E, Orvoëen-Frija E, Oppert JM, Huchon G. Left ventricular systolic dysfunction in patients with obstructive sleep apnea syndrome. Chest 2002; 122: 1133-1138

10 Pepperell J, Ramdassingh-Dow S, Crosthwaite N et al. Ambulatory blood pressure after therapeutic and subtherapeutic nasal continuous positive airway pressure for obstructive sleep apnoea: a randomised parallel trial. Lancet 2002; 359: 204-210

11 Randerath WJ, Schraeder O, Galetke W, Feldmeyer F, Ruhle KH. Autoadjusting CPAP therapy based on impedance: efficacy, compliance and acceptance. Am J Respir Crit Care Med 2001; 163: 652-657

12 Rasche K, Orth M, Duchna HW, Ullrich HU, Schultze-Werninghaus G. CPAP-Therapie bei obstruktivem Schlafapnoesyndrom. Nebenwirkungen und Akzeptanz im Langzeit-Verlauf. Med Klin 1994; 89: 86-88

${ }^{13}$ Reeves-Hoche MK, Hudgel DW, Meck R, Witteman R, Ross A, Zwillich $\mathrm{CW}$. Continuous versus bilevel positive airway pressure for obstructive sleep apnea. Am J Respir Crit Care Med 1995; 151: 443-449

${ }^{14}$ Sanner B, Heise M, Hinz R, Kopp J. Therapie der obstruktiven Schlafapnoe mit intraoralen Schnarch-Therapie-Geräten. Somnologie 1999; 3: 62-66

15 Sanner B, Klewer J, Trumm A, Randerath W, Kreuzer I, Zidek W. Longterm treatment with continuous positive airway pressure improves quality of life in obstructive sleep apnoea syndrome. Eur Respir J 2000; 16: $118-122$

${ }^{16}$ Sanner B, Tepel M, Markmann A, Zidek W. Effect of CPAP therapy on 24hour blood pressure in patients with obstructive sleep apnea syndrome. Am J Hypertens 2002; 15: 251-257

17 Sullivan CE, Issa FG, Berthon-Jones M, Eves L. Reserval of obstructive sleep apnoea by continuous positive airway pressure through the nares. Lancet 1981; I: 862-865

18 Teschler H, Farhat AA, Exner V, Konietzko N, Berthon-Jones M. AutoSet nasal CPAP titration: constancy of pressure, compliance and effectiveness at 8 month follow-up. Eur Respir J 1997; 10: 2073-2078 